\title{
Time-Dependent Dirac Equation with Relativistic Mean-Field Dynamics Applied to Heavy-Ion Scattering
}

\author{
R. Y. Cusson \\ Gesellschaft für Schwerionenforschung, D-6100 Darmstadt, Germany, and Department of Physics, \\ Duke University, Durham, North Carolina $27706^{(a)}$ \\ P. G. Reinhard \\ Institut für Theoretische Physik, Universität Erlangen, D-8520 Erlangen, Germany
}

\section{J. J. Molitoris and H. Stöcker}

National Superconducting Cyclotron Laboratory and Department of Physics and Astronomy, Michigan State University, East Lansing, Michigan 48824, and Institut für Theoretische Physik der Johann Wolfgang Goethe Universität, D-6000 Frankfurt am Main, Germany (a)

M. R. Strayer

Oak Ridge National Laboratory, Oak Ridge, Tennessee 37831

and

W. Greiner

Institut für Theoretische Physik der Johann Wolfgang Goethe Universität, D-6000 Frankfurt am Main, Germany (Received 10 December 1984; revised manuscript received 6 September 1985)

We treat the relativistic propagation of nucleons coupled to scalar-and vector-meson fields in a mean-field approximation. The time-dependent Dirac and mean-meson-field equations are solved numerically in three dimensions. Collisions of ${ }^{16} \mathrm{O}(300,600$, and $1200 \mathrm{MeV} /$ nucleon $)+{ }^{16} \mathrm{O}$ are studied for various impact parameters. The results are compared to other recent theoretical approaches. The calculations predict spallation, large transverse-momentum transfer, and positiveangle sidewards flow, in qualitative agreement with the data in this energy regime.

PACS numbers: $25.70 . \mathrm{Np}, 24.10 .-\mathrm{i}$

The time-dependent Hartree-Fock (TDHF) theory is the most widely used microscopic description of low-energy heavy-ion collisions. ${ }^{1}$ However, for heavy-ion collisions at energies comparable to the nucleon mass, the TDHF method breaks down. ${ }^{2}$ Thus a variety of semiclassical models have been applied to understand the collision phenomena, e.g., the cascade model ${ }^{3}$ nuclear fluid dynamics ${ }^{4}$ and the VlasovUehling-Uhlenbeck (VUU) approach. ${ }^{5}$ However, the complicated dynamics at these energies is still not very well understood on a microscopic level.

In this Letter we employ a relativistic fieldtheoretical model to heavy-ion reactions, the timedependent Dirac equation (TDDE) with meanmeson-field dynamics. The model has been successfully applied to describe static properties of spherical nuclei and dense nuclear matter., 6 We consider a baryon field (nucleon), a scalar-meson field $(\Phi)$, and a vector-meson field $\left(V_{\mu}\right)$ interacting via a Lorentzinvariant local Lagrangean density (in units with $\hbar=c=1$ ),

$$
\mathscr{L}=\mathscr{L}_{\text {nuclear }}^{\text {free }}+\mathscr{L}_{\text {mesons }}^{\text {free }}-g_{S} \Phi \bar{\psi} \psi-g_{V} \bar{\psi} \gamma_{\nu} V^{\nu} \psi,
$$

where $F_{\mu \nu}=\partial_{\nu} V_{\mu}-\partial_{\mu} V_{\nu}$. We follow the notation and conventions of Bjorken and Drell. ${ }^{8}$ The parameters of the Lagrangean, Eq. (1), are given in Ref. 6. Although some nuclear properties may be dependent on modifications and refinements of this interaction, ${ }^{9}$ it represents a reasonable starting point for studying the bulk and single-particle properties of nuclei.

In the mean-field approximation the following equations of motion emerge from the Lagrangean density, Eq. (1):

$$
\begin{aligned}
& i \frac{\partial}{\partial t} \Psi=\left[-i \gamma_{0} \gamma \cdot \nabla+M_{B} \gamma_{0}\right.+g_{S} \gamma_{0} \Phi \\
&\left.+g_{V} \gamma_{0} \gamma_{\mu} V^{\mu}\right] \Psi, \\
&-\frac{\partial^{2}}{\partial t^{2}} \Psi=\left[-\nabla^{2}+M_{S}^{2}\right] \Phi+g_{S}\langle\bar{\Psi} \Psi\rangle, \\
&-\frac{\partial^{2}}{\partial t^{2}} V_{\mu}=\left[-\nabla^{2}+M_{V}^{2}\right] V_{\mu}+g_{V}\left\langle\bar{\Psi} \gamma_{\mu} \Psi\right\rangle,
\end{aligned}
$$

The gauge and continuity equations $\partial_{\mu} V^{\mu}=0$ and $\partial_{\mu}\left\langle\bar{\Psi} \gamma^{\mu} \Psi\right\rangle=0$ are used to obtain Eq. (2c). In the mean-field approximation the Eqs. (2b) and (2c) propagate classical meson fields. Equation (2a) is reduced to an equation for a single-nucleon wave function 
$\psi_{\alpha}(t)$ by an expansion of the baryon-field operator in a complete set of time-dependent states, $\Psi(t)$ $=\Sigma_{\alpha} \psi_{\alpha}(t) a_{\alpha}$, where $a_{\alpha}$ is the annihilation operator for a nucleon in the state $\alpha$. Details relating to these equations and the method of solution will be given elsewhere. ${ }^{10}$

We represent the meson fields and the baryon wave functions on a three-dimensional $(24 \times 24 \times 24)$ mesh in both coordinate and momentum space, with a mesh spacing of $0.5 \mathrm{fm}$ in coordinate space. Quantities in momentum space are obtained by means of a fast Fourier transform method. The numerical solution of Eqs. (2) is carried out with a variable-order predictorcorrector, ${ }^{11}$ which permits larger time steps during the evolution $\left(\Delta t-\frac{1}{20} \mathrm{fm} / c\right)$ than in a previous work in one dimension. ${ }^{12}$ We employ isospin-degenerate nucleon wave functions and treat the spin degrees of freedom in the usual four-component spinor formalism. Thus we propagate eight independent fourcomponent wave functions for each ${ }^{16} \mathrm{O}$ nucleus.

We use the relativistic static Hartree wave functions for each of the ${ }^{16} \mathrm{O}$ initial states. The nuclei are located at a separation distance of $10 \mathrm{fm}$ from each other on the mesh, and are Lorentz boosted to the appropriate initial energy in the center-of-velocity frame. The time evolution for the baryon wave functions is done with a fifth-order predictor-corrector in momentum space and the meson equations are solved simultaneously by Green's functions techniques. With the above mesh, the momentum-space truncation occurs at about $2 \mathrm{GeV} / c$. As a test of the accuracy of our numerical techniques we evaluate the total energy at each time step in the evolution, and note that it is constant

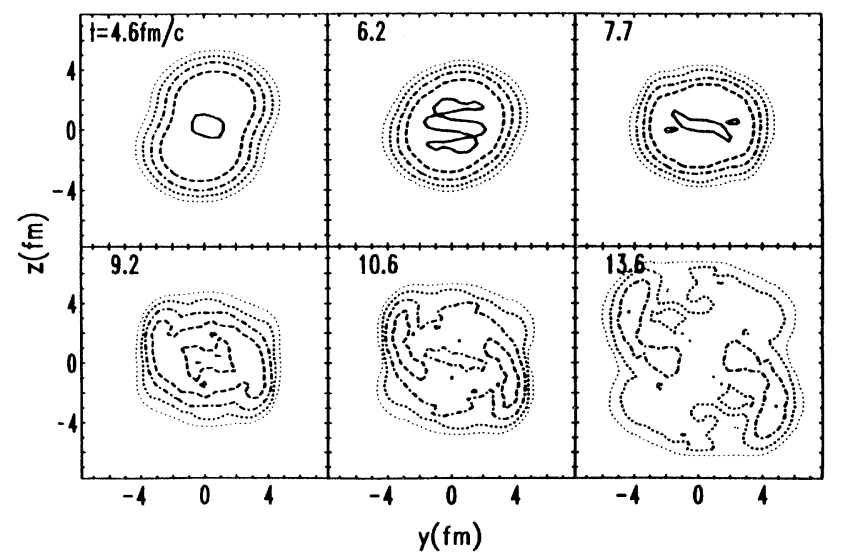

FIG. 1. Countour snapshots of the coordinate-space baryon density $\rho_{B}(x=0, y, z, t)$, in the scattering plane for ${ }^{16} \mathrm{O}(600 \mathrm{~A} \mathrm{MeV}, b=2 \mathrm{fm})+{ }^{16} \mathrm{O}$. The maximum density is $0.29 \mathrm{fm}^{-3}$ and is attained at $6 \mathrm{fm} / c$. The values of the density contours are as follows: solid curve, $0.26 \mathrm{fm}^{-3}$; dashed curve, $0.10 \mathrm{fm}^{-3}$; dot-dashed curve, $0.04 \mathrm{fm}^{-3}$; large-dotted curve, $0.014 \mathrm{fm}^{-3}$; and small-dotted curve, $0.005 \mathrm{fm}^{-3}$. to better than $1 \times 10^{-5}$. We have also evaluated the gauge and continuity conditions at each point in time to further guarantee the correctness of our solutions.

We have studied with this TDDE approach collisions of ${ }^{16} \mathrm{O}\left(E_{\mathrm{lab}}=300,600\right.$, and $1200 \mathrm{MeV} /$ nucleon $)+{ }^{16} \mathrm{O}$ at various impact parameters. The time evolution is followed until the expanding matter reaches the edges of the mesh. We show in Fig. 1 the time evolution of the spatial baryon density for $E_{\text {lab }}=600 \mathrm{MeV} /$ nucleon and $b=2 \mathrm{fm}$. The early-time behavior in the TDDE approach is similar to that obtained by use of nonrelativistic TDHF. Subsequently, a compression zone and sidewards flow develop (between $t=6$ and $10 \mathrm{fm} / c$ ), after which the system proceeds to undergo spallation. This result can be contrasted with TDHF calculations at medium energies where projectilelike and targetlike fragments emerge after the reaction. ${ }^{11}$ The sidewards flow observed in the present approach resembles the large transverse-momentum transfer predicted by nuclear fluid dynamics ${ }^{4}$ and the VUU approach. ${ }^{5}$

The large transverse-momentum transfer can be seen directly in Fig. 2, which shows-for the same reaction as displayed in Fig. 1-the corresponding time dependence of the momentum-space projection of the baryon density. It is evident that the transversemomentum transfer occurs mainly between 6.2 and $9.1 \mathrm{fm} / c$ : The momentum-space distribution has reached its asymptotic value by then and does not change during the subsequent expansion noted in Fig. 1.

It can be seen that the mid rapidity (center-of-mass) region is rather weakly populated after the collision; the population of the zero-c.m.-momentum states be-

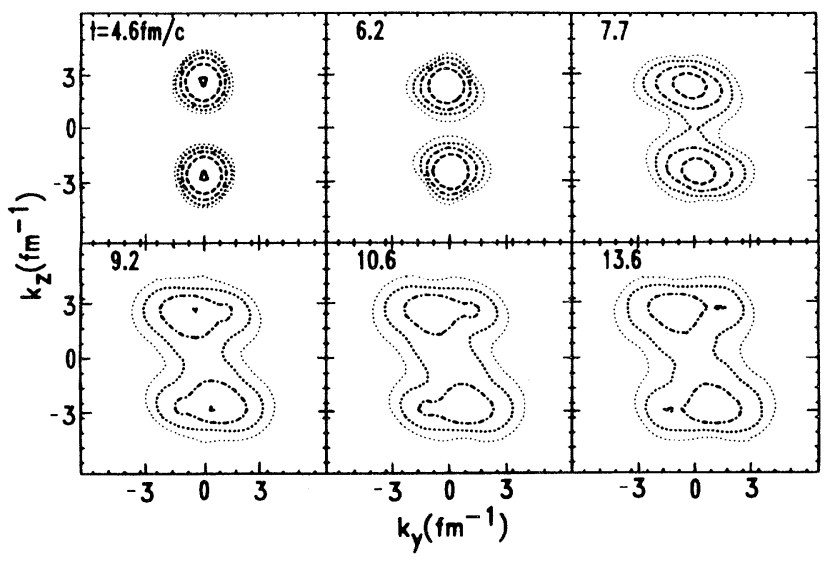

FIG. 2. The corresponding contours of the momentumspace baryon density, integrated over the component of the momentum perpendicular to the collision plane, $\rho_{B}(x$ $\left.=0, k_{y}, k_{z}, t\right)$. The initial beam momentum is along the $k_{z}$ axis. The values of the contours are solid lines, $0.90 \mathrm{fm}^{-1}$; dashed lines, $0.34 \mathrm{fm}^{-1}$; dot-dashed lines, $0.13 \mathrm{fm}^{-1}$; largedotted lines, $0.05 \mathrm{fm}^{-1}$; and small-dotted lines, $0.02 \mathrm{fm}^{-1}$. 
comes more complete at $300 \mathrm{MeV} /$ nucleon and is even less pronounced at $1200 \mathrm{MeV} /$ nucleon. This partial filling of the zero-momentum states can be contrasted with hydrodynamic calculations ${ }^{4}$ and VUUequation results ${ }^{5}$ where complete filling of the mid rapidity region is predicted. Experiments ${ }^{13}$ with heavier systems at 400 and $800 \mathrm{MeV} /$ nucleon suggest that more particles do indeed fill the zero-momentum region than predicted by our calculation. The partial filling seen in the present work could be a result of the particularly light system considered. Since the interaction time is shorter for the light-mass systems, the damping of the longitudinal momenta via the scattering is correspondingly low. We have also performed VUU calculations and observe, in contrast to the TDDE results, a plateau at the mid rapidity region even for the rather light system considered here.

In Fig. 3 we show the longitudinal-momentum dependence of the mean transverse momentum, as projected into the reaction plane ${ }^{5,14}$ The transversemomentum transfer represents a sensitive measure of the sidewards flow. The pronounced peak structure in the projectile and target rapidity regions is in qualitativer agreement with recent experimental results obtained from a streamer chamber for the system $\operatorname{Ar}(1.8$ $\mathrm{GeV} /$ nucleon) $+\mathrm{KCl}$ (Ref. 14) and plastic-ball-detector data for $\mathrm{Nb}(0.4 \mathrm{GeV} /$ nucleon) $+\mathrm{Nb}$ (Ref. 13).

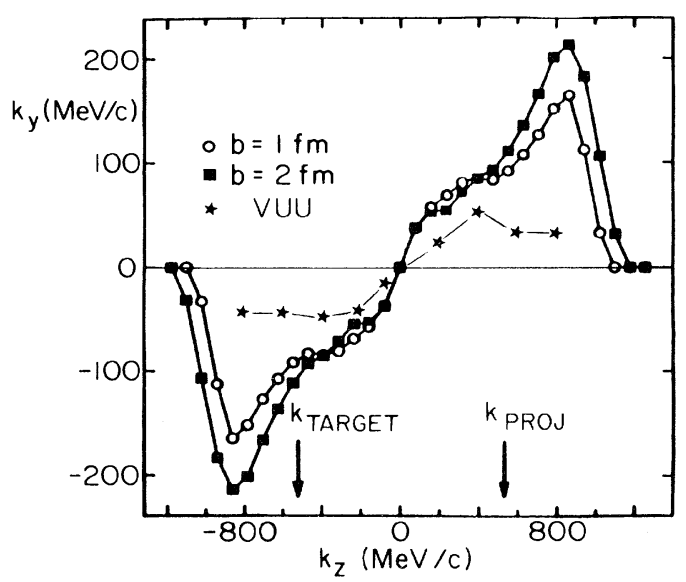

FIG. 3. The transverse momentum projected into the reaction plane and averaged over all perpendicular momenta, $k_{y}$, as a function of the longitudinal momentum, $k_{z}$, for the ${ }^{16} \mathrm{O}+{ }^{16} \mathrm{O}$ collision at an energy of $600 \mathrm{MeV} /$ nucleon. The circles denote the $b=1-\mathrm{fm}$ result and correspond to a mean flow angle of $9^{\circ}$, and the solid squares denote the $b=2-\mathrm{fm}$ result and correspond to a mean flow angle of $16^{\circ}$. The stars present the results of the corresponding VUU calculation with a stiff nuclear equation of state, $K=380$ $\mathrm{MeV} /$ nucleon, which has been shown to best reproduce the experimental data on pion production, flow angular distributions, and transverse-momentum distributions in heavier systems (Ref. 5).
TDDE calculations for these heavier systems would be highly desirable at this point to allow for a quantitative comparison with these data. However, about $100 \mathrm{~h}$ of central-processing-unit time have been spent on an IBM 3083 processor for the calculations presented above and the time required increases as the square of A.

In order to get a feeling for the significance of the absolute magnitude of the transverse-momentum transfer predicted in the TDDE approach, we have calculated $k_{y}$ vs $k_{z}$ for the above $\mathrm{O}+\mathrm{O}$ system in a variety of other current models: We used the standard nonrelativistic TDHF model ${ }^{11}$ and the recently presented three-dimensional Vlasov-equation solution, ${ }^{5}$ which is the classical analog to TDHF. We find that $k_{y}$ is compatible with zero (within the numerical uncertainties) for both approaches, even if a stiff compression potential is used. Also our cascade calculations result in very small transverse momenta $p_{y}\left(p_{z}\right)$, in accord with the results for heavier systems. ${ }^{5,13}$ Finite transverse-momentum transfers are predicted by the VUU approach ${ }^{5}$ and it has recently been shown ${ }^{5}$ that the absolute magnitude of the transverse momenta is linearly proportional to the stiffness of the nuclear compression potential. The results of the VUU calculation with a stiff compression potential $(K=380 \mathrm{MeV})^{5}$ are shown in Fig. 3 (stars) for the present system to allow for a direct comparison to our TDDE results (with $K=550 \mathrm{MeV}^{6,7}$ ). The momentum transfers predicted by the TDDE approach presented here do indeed exceed the VUU results considerably. A detailed study of the influence of the incompressibility in the TDDE approach is presently under way. ${ }^{10}$

It is interesting to note how the collective flow arises in the present mean-field theory. We find that the scalar and vector mean fields develop strong out-ofphase oscillations in space and time, soon after the maximum compression point. The corresponding large change in field energy is, of course, compensated elsewhere and the total energy remains conserved. The nucleons are deflected by these oscillations in the fields, giving rise to the sidewards flow.

We have computed relativistic collisions of ${ }^{16} \mathrm{O}+{ }^{16} \mathrm{O}$ in a relativistic mean-field model with scalar and vector mesons coupled to a baryon field. The TDDE results show spallation and collective sidewards flow, in agreement with the general features of the experimental data in the gigaelectronvolt-per-nucleon bombarding-energy range. In contrast to these data our calculations yield significantly fewer spallated nucleons near mid rapidity. The meson fields develop strong oscillations which cause the transverse-momentum transfer and the breakup. These results raise a number of interesting theoretical questions: The inclusion of nonlinear terms for the $\sigma$ field and other 
mesons ${ }^{7}$ could strongly influence the flow properties; explicit two-nucleon interaction terms which are omitted in the mean-field approximation may play an important role in understanding heavy-ion collisions at relativistic energies.

This work was supported by Gesellschaft für Schwerionenforschung, Darmstadt, Germany, the Bundesministerium für Forschung und Technologie, Bonn, Germany, and the U.S. Department of Energy under Contracts No. DE-AC05-84OR21400 and No. DE-A505-83ER40101.

(a) Permanent address.

1 Time Dependent Hartree-Fock and Beyond, edited by K. Goeke and P.-G. Reinhard, Lecture Notes in Physics, Vol. 171 (Springer-Verlag, Berlin, 1982).

${ }^{2}$ K. T. R. Davies, K. R. S. Devi, S. E. Koonin, and M. R. Strayer, in Treatise on Heavy Ion Science, edited by D. A. Bromley (Plenum, New York, 1985), Vol. 3.

${ }^{3}$ J. Cugnon, T. Mizutani, and J. Vandermeulen, Nucl. Phys. A352, 505 (1980).

${ }^{4}$ H. Stöcker, J. Maruhn, and W. Greiner, Phys. Rev. Lett. 44, 725 (1980); G. Buchwald, G. Graebner, J. Theis, J. Maruhn, W. Greiner, and H. Stöcker, Phys. Rev. Lett. 52, 1954 (1984).
${ }^{5}$ H. Kruse, B. Jacak, and H. Stöcker, Phys. Rev. Lett. 54, 289 (1985); J. J. Molitoris and H. Stöcker, Phys. Rev. C 32, 346 (1985).

6J. D. Walecka, Ann. Phys. (N.Y.) 83, 491 (1974); B. D. Serot and J. D. Walecka, Annu. Rev. Nucl. Sci. (to be published).

7J. Boguta and A. R. Bodmer, Nucl. Phys. A292, 414 (1977); J. Boguta and H. Stöcker, Phys. Lett. 120B, 289 (1983).

8J. D. Bjorken and S. D. Drell, Relativistic Quantum Field Theory (McGraw-Hill, New York, 1965).

${ }^{9}$ M. R. Anastasio, L. S. Celenza, W. S. Pong, and C. M. Shakin, Phys. Rep. 100, 327 (1983).

${ }_{10}$ R. Y. Cusson, P. G. Reinhard, H. Stöcker, M. R. Strayer, and W. Greiner, to be published.

${ }^{11}$ H. Stöcker, R. Y. Cusson, J. Maruhn, and W. Greiner, Z. Phys. A 294, 125 (1980).

${ }^{12}$ K. H. Müller, Nucl. Phys. A372, 459 (1981).

${ }^{13}$ H. A. Gustafsson, H. H. Gutbrod, B. Kolb, H. Löhner, B. Ludewigt, A. M. Poskanzer, T. Renner, H. Reidesel, H. G. Ritter, A. Warwick, F. Weik, and H. Weiman, Phys. Rev. Lett. 52, 1590 (1984); R. E. Renford, D. Schall, R. Bock, R. Brockmann, J. W. Harris, A. Sandoval, R. Stock, H. Stroebele, D. Bangert, W. Rauch, G. Odyniec, H. G. Pugh, and L. S. Schroeder, Phys. Rev. Lett. 53, 763 (1984).

${ }^{14}$ P. Danielewicz and G. Odyniec, Phys. Lett. 129B, 283 (1985). 